\title{
The Application and Research of NI Controller on Cold-rolling Simulator
}

\section{Ce Wang ${ }^{a}$, Ziying Liu ${ }^{b}$, Bingkui Wang ${ }^{c}$, Changli Zhang ${ }^{d}$, Xudong $\mathrm{Li}^{\mathrm{e}}$ and Bo Gong ${ }^{f}$}

\author{
Shougang Research Institute of Technology, Beijing 100043, China. \\ awangce@shougang.com, bzoonlzy@163.com, 'wangbingkui456@163.com, \\ dzhangchangli@shougang.com, lixudonggo@163.com, fgongbo@shougang.com
}

Keywords: Cold-rolling simulator, Electric control system, NI PXI Controller, Siemens PLC.

\begin{abstract}
This cold-rolling simulator is mainly used for exploitation of new productions and research for new technique. The execution system of this cold-rolling simulator includes hydraulic drive system and electric drive system. The features, such as multivariable, nonlinear, real-time, and high-speed, set high requirements for the control system. The PXI system is a compact modular PC platform for test, measurement, and control system, which meets the specific needs of the cold-rolling simulator by adding an integrated trigger bus and reference clock for multiple-board synchronization, a star trigger bus for very precise timing, and local buses for side-band communication. This control system of the cold-rolling simulator is mainly composed of PXI system and S7 PLC.
\end{abstract}

\section{Introduction}

This cold-rolling simulator is mainly used for exploitation of new productions including steel for automobiles: DDQ-HSS, BH-HSS, DP-HSS, TRIP-HSS, Twip and electrical steel: high performance oriented or non-oriented electric steel, and research for new technique. The cold-rolling simulator can achieve the research for the roughness of cold rolling sheet, technical lubricating, reduction, the relationship between texture and structure property, skin passing technique, etc. The control difficulty of this system lies in the parts of HGC cylinders and the tension cylinders. The hydraulic servo system with high frequency response and large output torque requires high-speed real-time closed-loop control, for example electro-hydraulic servo position control, electro-hydraulic servo pressure control, tension control and flow control.

In order to solve the problem of the system features of the experimental equipment, we designed the system architecture of the NI PXI controller and Siemens PLC. The NI PXI/CompactPCI embedded computer is a high-performance PXI/CompactPCI system controller, which integrates standard I/O features in a single unit by using state-of-the-art packaging and expand communication board, such as Profibus-DP bus or CAN bus. The Siemens PLC is responsible for the start-stop control, signal detection and auxiliary system control for the pump station.

\section{Development of System}

\subsection{Cold-rolling Simulator.}

The cold-rolling simulator consists of a mill stand, pressure reduction cylinder, tension cylinder, pull-back cylinder, hydraulic system and electric drive system. The mill stand is a four-post rack, with servo cylinders loaded between the upper and lower beams of the loading rack. When the servo cylinder is extended, the rack is deformed to generate a load force on the cylinder. The tension cylinder and pull-back cylinder are responsible for pulling the strip at a certain speed and maintaining a certain tension. The working schematic is shown in the figure 1 below. The difficulty in control of this equipment lies in the electro-hydraulic servo control. In order to meet the system's high precision requirements for hydraulic cylinder position, pressure, and speed, the servo valve's frequency response of the hydraulic system is required to be fast enough and the output power is large enough. Servo control system has high frequency response (usually above 10HZ), large output force (up to 35 
$000 \mathrm{KN})$. High frequency response servo systems require control systems with higher response frequencies to meet demand.

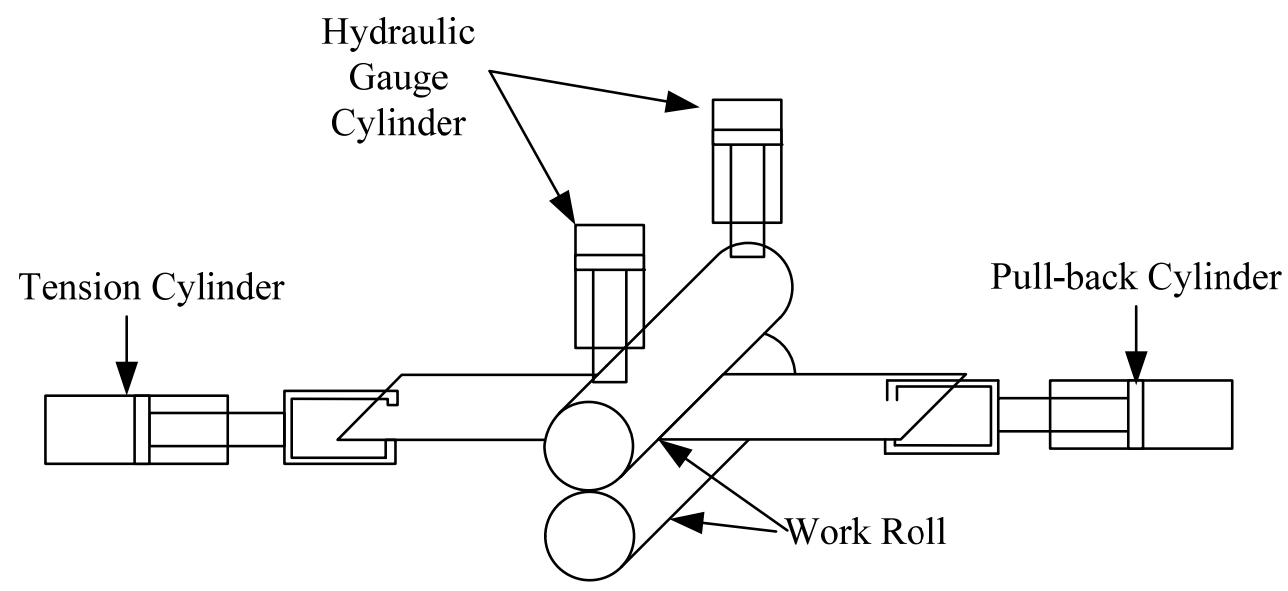

Fig 1. Working schematic of the cold-rolling simulator

\subsection{Control System.}

NI controllers for the control system use PXI-8106 RT controller, which can offer a high-performance platform, ideal for real-time test and control applications by LabVIEW application with the National Instruments LabVIEW Real-Time Module on Windows and downloading the program to the PXI-8106 RT controller via Ethernet. The NI 9203, which is a C Series DAQ module with 8 analog current input channels for high-performance control and monitoring applications, is used to acquire signals and output control signals. The module features programmable input ranges of $\pm 20 \mathrm{~mA}$ or $0 \mathrm{~mA}$ to $20 \mathrm{~mA}, 16$-bit resolution, and a $200 \mathrm{kS} / \mathrm{s}$ maximum sampling rate. For acquiring the position signal transferred by CAN bus, we chose the NI 9853 module, which has two 9-pin male D-Sub connections that provide connections to a CAN bus. These modules are installed on the expansion chassis NI 9151 installed on the main chassis NI PXI-1042. The control system block diagram as shown in figure 2 below.

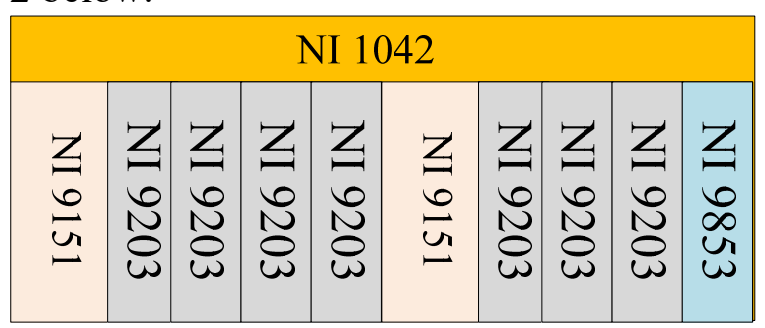

Fig 2. Control system block diagram

The siemens PLC for this system uses CPU315-2DP as the main station, hanging several ET200S slave stations including rolling drive station, hydraulic drives \& auxiliary drives station, hydraulic on-site station, ball valves inlet station, ball valves outlet station, mill stand station. The rolling drive station is mainly responsible for collecting main drive power supply, operating status, alarm and enable functions. The hydraulic drives \& auxiliary drives station is mainly responsible for checking the power supply status of these pumps including AGC pump, Control pump and Storage load pump. The hydraulic on-site station is mainly responsible for detecting the oil level, pressure, temperature of the pump station, and controlling the loading and unloading of each oil pump. The ball valve inlet station and ball valve outlet station are responsible for checking the limit switches of the tension cylinder, pull-back cylinder and accumulator. The mill stand station is responsible for checking the HGC cylinders' position, the status of the valves installed on the mill stand, and controlling the auxiliary cylinders, such as cutting off cylinder, control cylinder, adjustment cylinder, roller cylinder, etc.

In order to improve the integrity and reliability of the system, the program uses a variety of fieldbus communication methods, for example Profibus-DP, CAN bus, Ethernet, etc. PCs with communication boards that supports the Profibus-DP protocol communicate with CPU 315-2DP. The communication between master and slave stations also through Profibus-DP protocol. The position 
sensors, measuring the position of the HGC cylinders, communicate with the NI 9853 via CAN bus. The system communication diagram as shown in the figure 3 below.

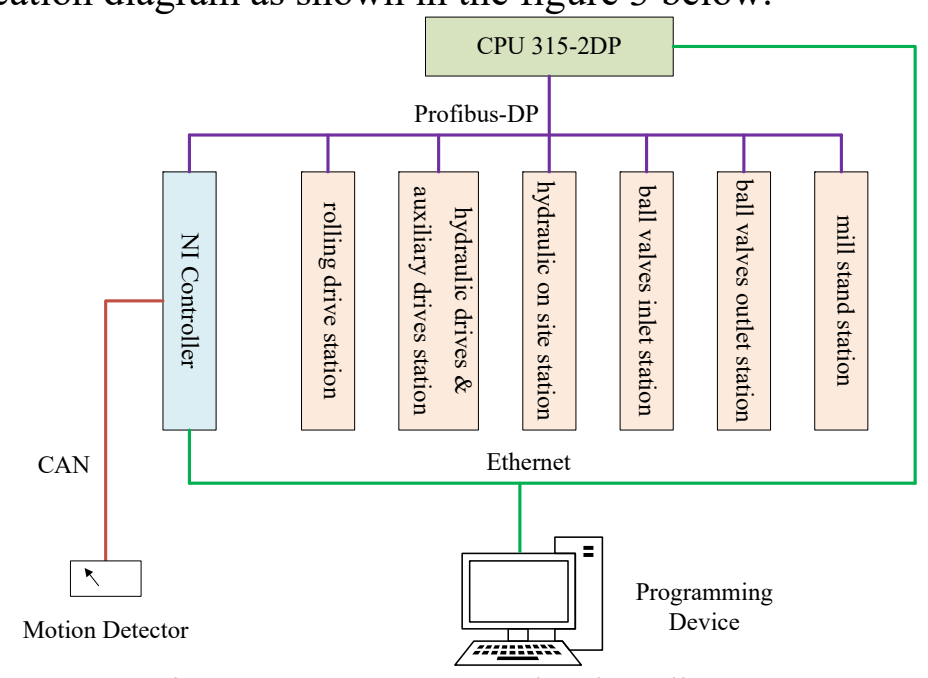

Fig 3. System communication diagram

\section{Application Effect}

The application program consists of two parts: FPGA program part and Real-Time part, developed through LabVIEW. The FPGA program runs on the cRIO FPGA chip. It is characterized by high-speed parallelism and can run multiple parallel loops. The speeds of each loop independently. We can set the parameters of each pass through the human machine interface, such as the amount of reduction, front and rear tension, speed and so on. We can also record various process data and display it through real-time curve and historical curve. The related operation interfaces are shown in the figure 4 below.

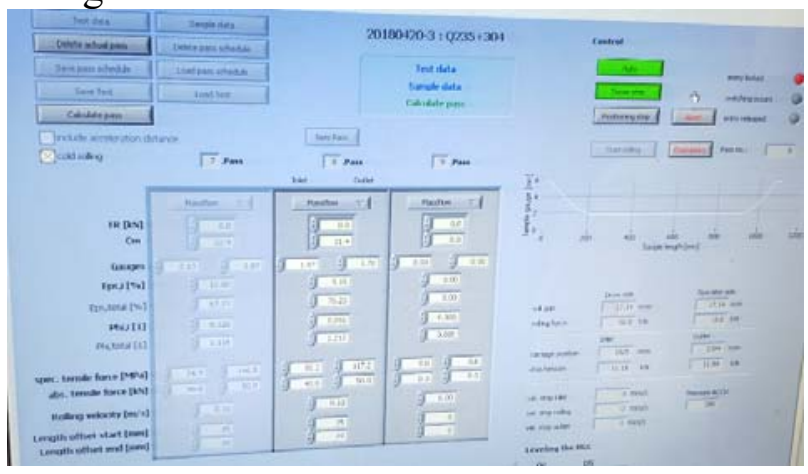

(a)Parameter setting interface

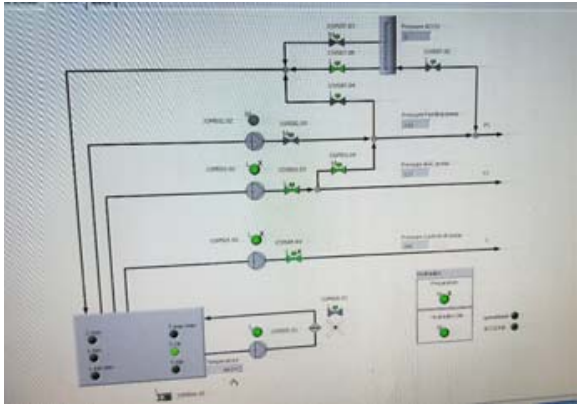

(c)Hydraulic monitoring interface

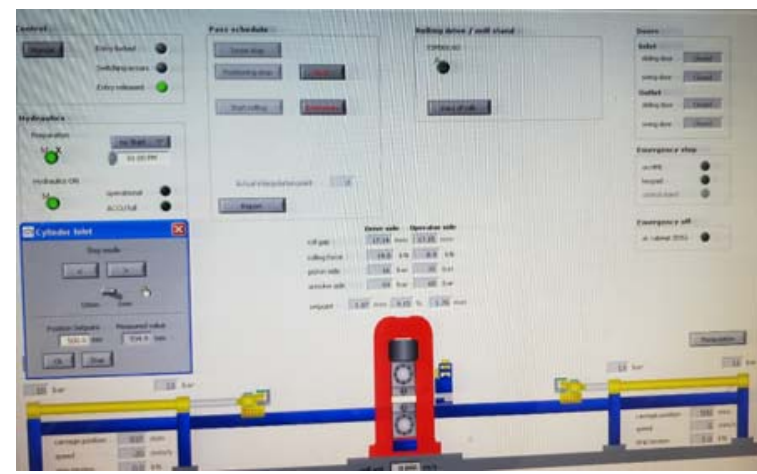

(b)Main monitoring interface

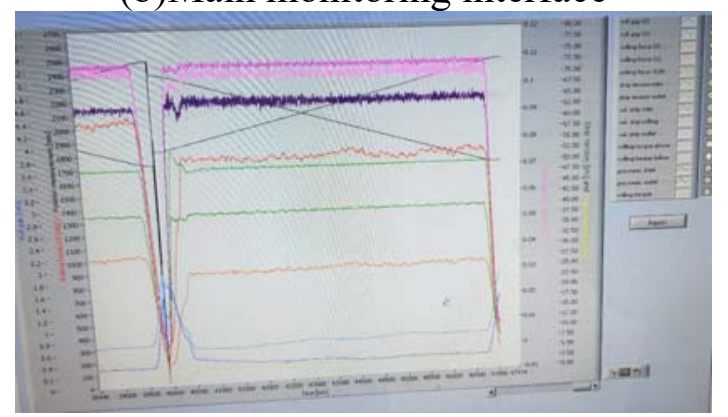

(d)History curve interface

Fig 4. Operation interface

\section{Summary}

Since the control system has been put into operation, it has been operating stably and meets various test conditions. In summary, this system has the following features: 
(1) With parallel running function, multiple closed-loop control algorithms can be run simultaneously with multiple CPUs.

(2) The core computing unit has a high operation speed and can satisfy closed-loop control algorithms requiring high-speed operating ability, such as PID or neural network control algorithms.

(3) Making full use of a variety of fieldbus communication methods, which greatly improves the system's interconnectivity, reliability, and make the system architecture more clearly.

(4) Using a variety of control systems in combination to maximize their respective functions.

\section{References}

[1]. Xiukun Ding, Rolling process automation, Metallurgical Industry Press, 2009, p. 57-167.

[2]. Yikang Sun, Strip Cold Rolling Computer Control, Metallurgical Industry Press, 2002, p. 1-201.

[3]. Shaoming Li, Longsong $\mathrm{Xu}$, Yue Hua, et al. Research on hydraulic AGC system based on GA fuzzy PID algorithm. USA. Proceeding of 2014 International Conference on Simulation and Modeling Methodologies, Technologies and Application, USA, 2014:9, p. 363-371.

[4]. Hongge Ren, Dongmei Li, et al. Active Learning Algorithm in The Application of Hydraulic AGC System. $201527^{\text {th }}$ Chinese Control and Decision Conference(CCDC), 2015, P. 530-535. 